\title{
Small bowel incarceration in the umbilical artery following total laparoscopic radical hysterectomy
}

\author{
Juan Gilabert-Estelles • Riccardo Favero • \\ Vicente Paya $\cdot$ Sergio Costa $\cdot$ Francisco Coloma • \\ Juan Gilabert-Aguilar
}

Received: 14 August 2009 / Accepted: 8 September 2009/Published online: 23 September 2009

(C) Springer-Verlag 2009

\begin{abstract}
Total laparoscopic radical hysterectomy (TLRH) has demonstrated to be a feasible and safe technique for patients affected of early cervical cancer. Small bowel obstruction resulting from a loop volvulus represents a very uncommon postoperative complication in gynecological laparoscopic surgery. We report a case of a patient who presented an intestinal obstruction following a TLRH for cervical cancer. The obstruction was caused by entrapment of a segment of small bowel under the dissected obliterated umbilical artery resulting in a loop incarceration. Wide radical pelvic dissection in radical hysterectomy usually leaves uncovered many dissected retroperitoneal structures. Postoperative bands and adhesions represent the main cause of bowel obstruction after a surgical procedure. Retroperitoneal vessel dissection is mandatory to achieve safely an adequate radicality, but it may lead to intestinal complications that should be taken into account. To our knowledge, this is the first report of postoperative bowel incarceration through the umbilical artery after a laparoscopic oncological procedure.
\end{abstract}

Keywords Radical hysterectomy · Laparoscopy · Small bowel incarceration . Volvulus

J. Gilabert-Estelles $(\bowtie)$

Department of Gynecology, La Fe University Hospital,

Avda. Campanar 2146009 Valencia, Spain

e-mail: juangilaeste@yahoo.es

R. Favero $\cdot$ V. Paya $\cdot$ S. Costa $\cdot$ F. Coloma $\cdot$ J. Gilabert-Aguilar Department of Gynecology, Hospital Arnau de Vilanova, Valencia, Spain

\section{Introduction}

During the last decades, laparoscopy is being widely accepted for gynecological surgical procedures due to minimal trauma for the patient and quick recovery with results comparable to classical laparotomic techniques. Several studies in the recent literature have demonstrated that TLRH with pelvic and aortic lymph node dissection is a feasible technique for patients affected of early cervical cancer (stages Ia2-Ib1), with comparable results to the traditional laparotomic approach. Laparoscopic surgery offers the potential benefits of reduced discomfort, short hospital stay and convalescence, small abdominal scar, and low morbidity [1-4]. Most frequently reported postoperative complications after TLRH are bladder and rectal voiding dysfunction, urinary tract infection, thromboembolic events, urinary fistula, cuff abscess, pelvic lymphocyst, and abdominal wall hematoma [1-4].

Volvulus as a cause of small bowel obstruction represents a very uncommon postoperative complication in advanced gynecological laparoscopic surgery. Meanwhile, it is a rare but well recognized complication following total abdominal hysterectomy in the presence of adhesions [5, 6]. Injury to the peritoneum and peritoneal ischemia during surgery predisposes to the formation of adhesion and bands, which are the main causes of bowel obstruction after a surgical procedure. The estimated prevalence of intraabdominal adhesions after laparotomy is being reported to be as high as $95 \%$. On the contrary, laparoscopic approach reduces the risk of postoperative adhesions, thus minimizing the incidence of complications related to this event $[5,6]$.

To our knowledge, this is the first reported case that describes small bowel obstruction due to a loop incarceration in the umbilical artery at the paravesical fossa after an 
oncological laparoscopic procedure. This case highlights one of the potential hazards of wide pelvic dissection, which is mandatory to achieve in order to obtain a proper radicality, but may lead to intestinal complications that should be taken into account.

\section{Case report}

A 40-year-old Caucasian nulligravid woman was referred to our cervical unit at Hospital Arnau de Vilanova (Valencia, Spain) for high-grade squamous intraepithelial lesion detected at a cytology exam. She was affected by Turner syndrome, and she did not have any previous surgery. Colposcopic examination of the cervix showed an abnormal pattern, and endocervical biopsy was positive for welldifferentiated adenocarcinoma. She underwent cervical conization resulting in well-differentiated infiltrative adenocarcinoma. The MRI showed a cervical mass sized $2.2 \times$ $1.7 \mathrm{~cm}$ with deep infiltration of the cervical wall and no infiltration of the parametrium. No lymph node metastases were detected. She was programmed for a piver type III [7] TLRH for Ib1 stage cervical cancer.

A four-trocar laparoscopic transperitoneal approach was used. Pelvic examination during laparoscopic surgery found atrophic uterus and ovaries, and no evident peritoneal implants. A type III [7] total laparoscopic radical hysterectomy with bilateral pelvic lymphadenectomy was performed similarly to previous reports by other groups [2, 4]. External, internal, interiliac, obturator, and common iliac lymph nodes were systematically removed. Laparoscopic approach to the retroperitoneum was facilitated by the wide dissection of the umbilical artery, which permitted an easy and blunt dissection of the paravesical and pararectal spaces. Mild traction of this structure facilitated the section of the paracervix at the pelvic wall. Final examination of the operative field showed the retroperitoneal anatomical landmarks (Fig. 1). The intraoperative and short-term early postoperative period was uneventful, with no complications. The operative time was $230 \mathrm{~min}$ with an estimated blood loss of $200 \mathrm{cc}$. No intraoperative blood transfusion was required. The pathologic examination confirmed that surgical margins of the specimen, parametria, and vagina were free of disease. No lymph node metastases of the pelvic lymphadenectomy were detected. Bowel activity was recovered $48 \mathrm{~h}$ after surgery, and the resumption of defecation occurred on the sixth day. Bladder training, using interval clamping of the urethral catheter, was started postoperatively. The patient was discharged uneventfully at the fifth day postoperatively. The bladder function was completely recovered, and the residual urine achieved to be negative on the 19th day.

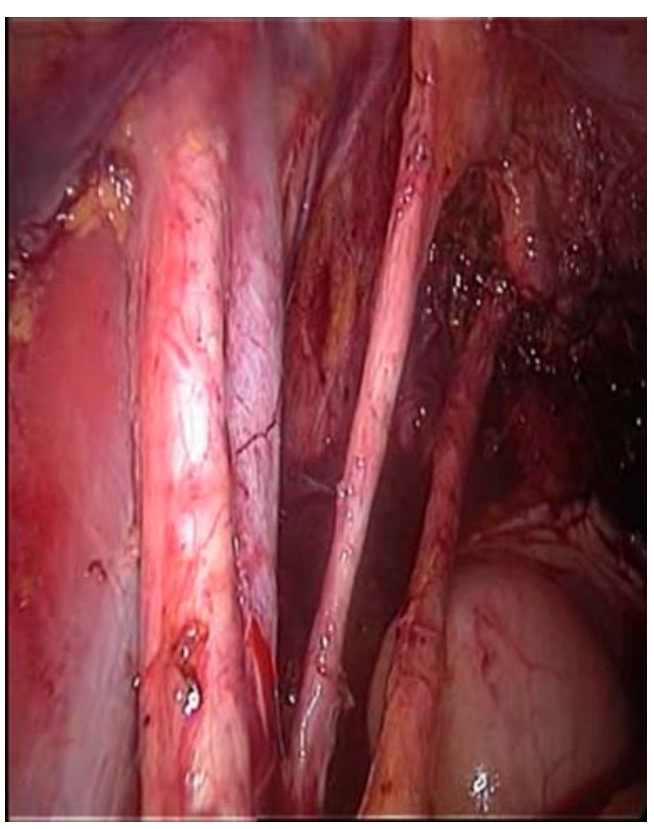

Fig. 1 Laparoscopic view of left retroperitoneal anatomy

On the 21th postoperative day, she was readmitted in the emergency area affected of acute abdominal pain since $2 \mathrm{~h}$, accompanied by nausea and vomiting. Clinically, she presented mucosal paleness, a painful abdomen on palpation, without any sign of peritoneal disease. Abdominal ultrasound examination revealed intestinal distension and moderate intraabdominal fluid. Abdominal X-ray detected the presence of dilated small bowel loops with air-fluid levels. TC scan was suggestive of a small bowel obstruction. A laparoscopy was initially performed in order to evaluate the abdominal cavity. Epiplon adhesions were found in the left pelvic side with the presence of free peritoneal serum-hematic fluid. Dissection of adhesions permitted the identification of an ischemic, almost gangrenous jejunal loop, due to a small bowel volvulus with mesenteric edema, venous engorgement, and bowel ischemia, but no peritoneal contamination (Fig. 2). General surgeon confirmed the absence of viability of the small bowel loop and the limited access due to bowel dilatation. A laparotomic conversion was therefore performed in order to clarify and solve the complication. The small bowel was examined carefully and followed up to the level of the vaginal cuff and the left umbilical artery. A fibrous vertical structure was identified to be the cause of bowel incarceration and volvulus formation of a jejunal loop. Careful ligation and section of this structure, initially suspected to be a fibrotic band, led to a complete release of the jejunum. Examination of the field identified the left obliterated umbilical artery, which was followed cranially, to be the real cause of the incarceration process. Macroscopic examination of the small bowel showed a segment of 


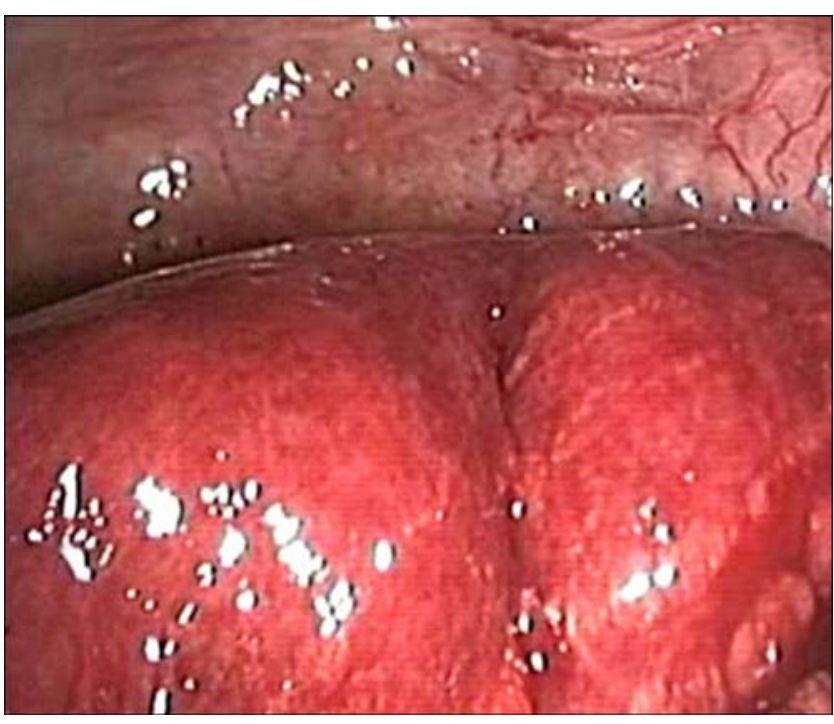

Fig. 2 Macroscopic appearance of bowel incarceration

$50 \mathrm{~cm}$ in length of ischemic intestine and a wide segmental bowel resection with end to end anastomosis was performed. The postoperative course was uneventful, and the patient was discharged home on the 11 th postoperative day.

\section{Discussion}

Small-bowel volvulus consists in an abnormal twisting of a loop of small bowel around the axis of its own mesentery, which produces a mechanical bowel obstruction. This process may also result in torsion and occlusion of the mesenteric vasculature, which can lead to bowel ischemia and final necrosis. Mortality for bowel volvulus has been estimated to be $9-35 \%$, but this may increase up to $20-100 \%$ in cases of extended bowel necrosis [8].

Radical hysterectomy with bilateral lymphadenectomy represents one of the most important strategies in the treatment of cervical cancer, especially in early stages of the disease with small tumor volume. Nowadays, 5-year survival rates of patients with stage IB and IIA range between $87 \%$ and $92 \%$ [9].

During past 10 years, laparoscopic approach has become increasingly important in gynecologic surgery. Acceptance of a new surgical technique in oncology requires that technical feasibility is demonstrated and that the morbidity and survival rates are equal to classical surgical approach $[2,3]$. In type III hysterectomy, a complete resection of the paracervix and uterosacral ligaments is performed and wide dissection and identification of structures at the retroperitoneum is mandatory in order to perform safe resection of the affected tissue. At our institution, a type III radicality is performed in cases of voluminous Ib1 cervical cancers measuring more than $2 \mathrm{~cm}$.
Early and late postoperative complications in TLRH have been reported to range from $9 \%$ to $27 \%$, being the most frequent ones, bladder dysfunction and urinary tract infection [1-3, 10]. Thromboembolic events, urinary fistula, cuff abscess, pelvic lymphocyst, and abdominal wall haematoma are infrequently reported (1-4\%). Obermair et al reported one case in their large series of small bowel obstruction that occurred 3 years after initial surgery for cervical cancer [1]. Intestinal complications are very infrequent after gynecological surgery and may be mainly due to the presence of postoperative adhesions $[5,6,11]$. Interestingly, Huntington et al reported a series of three cases of small bowel obstruction following laparoscopically assisted vaginal hysterectomy, resulting from extraneous retained staples used to divide the adnexa [12]. Laparoscopic surgery results in less invasive approach to cervical cancer that reduces the risk of postoperative adhesion formation [5, 6, 13, 14]. Meanwhile dissection is usually more meticulous. and retroperitoneal structures are usually completely dissected and exposed to the abdominal structures. In the present case, an anatomical retroperitoneal structure, such as the obliterated umbilical artery, and not a band or adhesion was the origin of incarceration of the bowel.

Identification and dissection of the obliterated umbilical artery is important during the TLRH procedure as it is a fundamental landmark during the dissection of the lateral pelvic space [15]. In addition, opening of the pararectal space is facilitated by the traction from the umbilical artery. Care must be taken if a tight and tense dissected umbilical artery is identified at the end of operation. In this situation, the section of the obliterated umbilical artery itself may avoid bowel incarceration, thus removing critical points, where a small bowel loop could wedge in. Meanwhile, surgical removal of retroperitoneal structures is only possible for nonfunctional structures, such as obliterated umbilical artery. Other options may be encountered to prevent this rare complication in any situation.

Postoperative adhesions might develop in up to $60-90 \%$ of patients undergoing gynecological surgery [13]. After the present report, our main concern has been the possibility of bowel volvulus in retroperitoneal anatomical structures. Agents for adhesion prevention may create an absorbable barrier that facilitate the regeneration of the peritoneum and prevent this complication. Application of solid agents, such as oxygenized regenerated cellulose (Interceed $^{\mathrm{TM}}$, Johnson\&Johnson, USA) or membrane polyD,L-lactide (PDLA), over the dissected retroperitoneal vascular structures might avoid mechanically the postoperative incarceration of the intestine. These barriers rapidly form a soft gelatinous mass that provides a protective coating around healing tissue during first days after application $[13,14]$. Other liquid agents, such as $4 \%$ icodextrin solution 
(Adept, Shire GmbH and Co. KG) or Hyalobarrier (Baxter $\mathrm{GmbH}$ ) may be useful in adhesion prevention but not as prevention of bowel incarceration [13].

In conclusion, in early cervical cancer, radical pelvic dissection is necessary in order to achieve a proper therapy. Even if small bowel incarceration represents a very rare event, the gynecological oncologist and the general surgeon must be aware of this possibility. It is important to take into account the possibility of late onset of the symptoms that might occur even after several weeks from radical pelvic surgery and the lifethreatening risks of misdiagnosing this entity.

Conflict of interest There is no actual or potential conflict of interest in relation to this article.

\section{References}

1. Obermair A, Ginbey P, McCartney AJ (2003) Feasibility and safety of total laparoscopic radical hysterectomy. J Am Assoc Gynecol Laparosc 10:345-349

2. Gil-Moreno A, Puig O, Perez-Benavente MA, Diaz B, Verges R, De la Torre J, Martinez-Palones JM, Xercavins J (2005) Total laparoscopic radical hysterectomy (type II-III) with pelvic lymphadenectomy in early invasive cervical cancer. J Minim Invasive Gynecol 12:113-120

3. Spirtos NM, Eisenkop SM, Schlaerth JB, Ballon SC (2002) Laparoscopic radical hysterectomy (type III) with aortic and pelvic lymphadenectomy in patients with stage I cervical cancer: surgical morbidity and intermediate follow-up. Am J Obstet Gynecol 187:340-348
4. Malzoni M, Tinelli R, Cosentino F, Perone C, Vicario V (2007) Feasibility, morbidity, and safety of total laparoscopic radical hysterectomy with lymphadenectomy: our experience. JMIG 14:584-590

5. Molloy KJ, Nasim A, Payne D, Lloyd DM (2000) Laparoscopic reduction of incarcerated Meckel's diverticulum following abdominal hysterectomy. JSLS 4:235-237

6. Al-Sunaidi M, Tulandi T (2006) Adhesion-related bowel obstruction after hysterectomy for benign conditions. Obstet Gynecol 108:1162-1166

7. Piver MS, Rutledge F, Smith JP (1974) Five classes of extended hysterectomy for women with cervical cancer. Obstet Gynecol 44:265-272

8. Katis PG, Dias SM (2004) Volvulus: a rare twist on small-bowel obstruction. CMAJ 171:728

9. Kolbl H (2001) Operative standard therapy of micro- and macroinvasive carcinoma of the cervix. Zentralbl Gynakol 123:237-244

10. Pomel C, Atallah D, Le Bouedec G, Rouzier R, Morice P, Castaigne D, Dauplat J (2003) Laparoscopic radical hysterectomy for invasive cervical cancer: 8-year experience of a pilot study. Gynecol Oncol 91:534-539

11. Al-Jaroudi D, Tulandi T (2004) Adhesion prevention in gynecologic surgery. Obstet Gynecol Surv 59:360-367

12. Huntington TR, Nishitani R, Belue JB, Klomp GR (1999) Small bowel obstruction secondary to stapled laparoscopically assisted vaginal hysterectomy. Surg Endosc 13:246-249

13. Wallwiener $M$, Brucker $S$, Hierlemann $H$, Brochhausen $C$, Solomayer E, Wallwiener C (2006) Innovative barriers for peritoneal adhesion prevention: liquid or solid? A rat uterine horn model. Fertil Steril 86:1266-1276

14. Johns A (2001) Evidence-based prevention of post-operative adhesions. Hum Reprod Update 7:577-579

15. Hatch KD (2001) Technique of schauta hysterectomy and laparoscopic lymphadenectomy. CME Journal of Gynecol Oncol 6:110-116 